\title{
Efficient preparation of azodye-labeled aminoxy acids and peptides
}

\author{
Suvendu Biswas, ${ }^{\text {a }}$ Srinivasa R. Tala, ${ }^{\text {a }}$ Akaki Kalatozishvili, ${ }^{\text {a,b }}$ and Alan R. Katritzky ${ }^{\text {a* }}$ \\ ${ }^{a}$ Center for Heterocyclic Compounds, Department of Chemistry, \\ University of Florida, Gainesville, FL 32611-7200, USA \\ ${ }^{b}$ Ivane Javakhishvili Tbilisi State University, 0128 Tbilisi, Georgia \\ E-mail: katritzky@chem.ufl.edu
}

This paper is dedicated to Professor Julio Álvarez-Builla, on the occasion of his $6^{\text {th }}$ anniversary

DOI: http://dx.doi.org/10.3998/ark.5550190.0012.317

\begin{abstract}
1-(4-Arylazobenzoyl)benzotriazoles $\mathbf{2 a}-\mathbf{c}$ react in aqueous acetonitrile at $20{ }^{\circ} \mathrm{C}$ with aminoxy acids 3a-c to give azodye-labeled aminoxy acids $\mathbf{4 a}-\mathbf{i}$ in $65-80 \%$ yields. Similarly, reaction of Fmocprotected aminoxy-dipeptides $\mathbf{6 a - c}$ with $2 \mathbf{2 a}$ gave azodye-labeled aminoxy peptides $\mathbf{7 a - c}$ in $55-65 \%$ yield.
\end{abstract}

Keywords: Azodyes, aminoxy acids, peptides, acylation

\section{Introduction}

Photoisomerization and fluorescence resonance energy transfer (FRET) properties have led to extensive use of azodye carboxylic acids such as $\mathbf{1 a}, \mathbf{1 b}$ and $\mathbf{1 c}$ (Figure 1) in biology. ${ }^{1-3}$ Azodye-labeled peptides are important for pharmaceutical and biological investigations (Figure 2). Peptide based azodye-labeled molecules show substrate specificity for prostate membrane antigen ${ }^{4}$ and in some cases are potent inhibitors of $m$-calpain. ${ }^{3}$ Azodye-labeled peptides may also serve as markers in biological applications. ${ }^{5}$

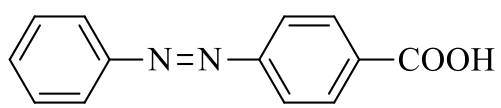

1a, 4-Paba = 4-(Phenylazo)benzoic acid

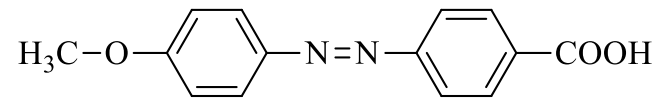

1b, 4-Mpaba = 4-(Methoxyphenylazo)benzoic acid<smiles>CN(C)c1ccc(N=Nc2ccc(C(=O)O)cc2)cc1</smiles>

1c, 4-Dpaba = 4-(Dimethylaminophenylazo)benzoic acid 
Figure 1. Azodye carboxylic acids.<smiles>CC(C)CC(C=O)NC(=O)[C@H](NC(=O)c1ccc(/N=N/c2ccccc2)cc1)C(C)C</smiles>

Potent inhibitor of m-calpain

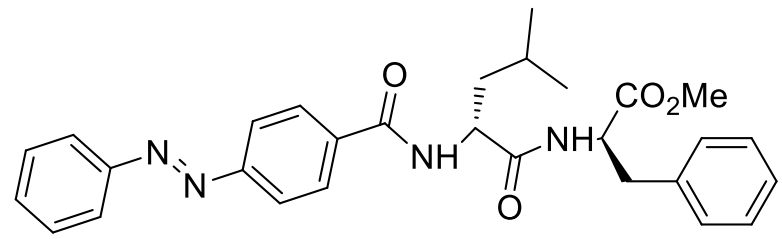

Potent inhibitor of alpha-chymotrypsin<smiles></smiles>

Peptide marker<smiles>COC(=O)C(=O)C(Cc1ccccc1)NC(=O)c1ccc(/N=N/c2ccccc2)cc1</smiles>

Photoswitchable inhibitor

Figure 2. Azodye labeled peptides.

Attaching azodye carboxylic acids to host molecules is the key to the synthesis of azophotoresponsive systems. Amino acids/peptides or amines acting as links between azo-dye acyl groups and host molecules are common in many photobiological switches and bioprobes. Azophotoresponsive systems frequently incorporate azodye-labeled $\alpha(\omega)$-amino acids/peptides. ${ }^{6}$

$\alpha$-Aminoxy acids are analogs of $\beta$-amino acids in which the $\beta$-carbon atom is replaced by an oxygen atom. The incorporation of $\alpha$-aminoxy acids into peptidomimetics has attracted interest, since $\alpha$-aminoxy acid units are more rigid than the corresponding $\beta$-amino acid units, ${ }^{7}$ and aminoxy

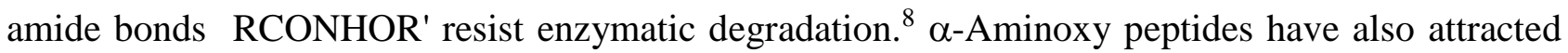
interest as novel foldamers ${ }^{9}$ with unusual conformations and diverse bioactivity. ${ }^{10 a-c}$ Aminoxy peptides also feature strong intramolecular hydrogen bonds between adjacent residues in peptidomimetic foldamers ${ }^{11}$ and may provide useful labels.

$\mathrm{N}$-Acylbenzotriazoles are easily prepared, non-hygroscopic, chirally stable analogs of acid halides that are relatively insensitive to water, ${ }^{12,13}$ and are therefore advantageous for N-, O-, C-, or S- acylation; ${ }^{12-18}$ especially where the corresponding acid chlorides are unstable or difficult to prepare. ${ }^{19,20}$ We previously acylated amino acids and amines with $N$-(4-arylazobenzoyl)- $1 H$ benzotriazoles $^{21}$ and we recently synthesized azodye-labeled peptides in good yields by a milder procedure than published methods. ${ }^{22}$

We have not located any previous syntheses of azodye-labeled aminoxy acids or peptides. Because of the interesting properties of aminoxy peptides, we now report the synthesis of azodye 
labeled aminoxy acids and peptides by reaction of $\mathrm{N}$-(4-arylazobenzoyl)- $1 H$-benzotriazole with aminoxy acids and aminoxy peptides under mild conditions.

\section{Results and Discussion}

\section{Preparation of azodye labeled aminoxy acids $(4 \mathbf{a}-\mathbf{i})$}

$N$-(4-Arylazobenzoyl)-1 $H$-benzotriazoles $\mathbf{2 a}-\mathbf{c}$, which were prepared by our previously reported method, ${ }^{21 b}$ were treated with the appropriate aminoxy acids $3 \mathbf{a}-\mathbf{c}$ in $\mathrm{THF}-\mathrm{H}_{2} \mathrm{O}(3-1, \mathrm{v} / \mathrm{v})$ in the presence of triethylamine for $4-8 \mathrm{~h}$ at $20{ }^{\circ} \mathrm{C}$ (monitored by TLC) to afford azodye labeled aminoxy acids $\mathbf{4 a}-\mathbf{i}$ in yields of $65-80 \%$ (Scheme 1, Table 1). Novel products were characterized by ${ }^{1} \mathrm{H}-\mathrm{NMR},{ }^{13} \mathrm{C}-\mathrm{NMR}$ and elemental analysis.

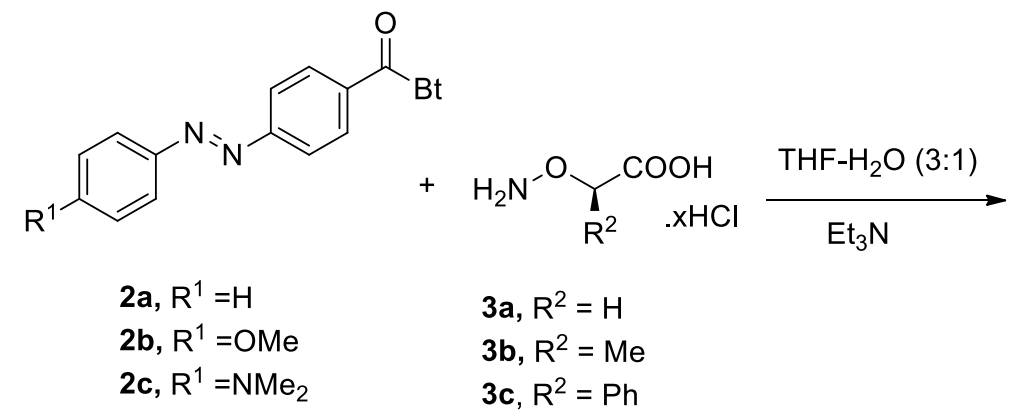<smiles>[R]c1ccc(/N=N/c2ccc(C(=O)NOC([R2])C(=O)O)cc2)cc1</smiles>

$4 a-i, 65-80 \%$

\section{Scheme 1}

Table 1. Preparation of azodye carboxylic acid labeled aminoxy acids $4 \mathbf{a}-\mathbf{i}$

\begin{tabular}{ccccc}
\hline Entry & $\mathbf{2}$ & Aminoxy acid 3a & 4, yield $(\%)^{\mathrm{b}}$ & $\mathbf{4 ,}$, ${ }^{\mathrm{a}}{ }^{\circ} \mathrm{C}$ \\
\hline 1 & $\mathbf{2 a}$ & AO-Gly-OH 3a & 4-Paba-AO-Gly-OH 4a, 74 & $200-202$ \\
2 & $\mathbf{2 a}$ & AO-Ala-OH 3b & 4-Paba-AO-Ala-OH 4b, 75 & $188-190$ \\
3 & $\mathbf{2 a}$ & AO-Phe-OH 3c & 4-Paba-AO-Phe-OH 4c, 70 & $178-180$ \\
4 & $\mathbf{2 b}$ & AO-Gly-OH 3a & 4-Mpaba-AO-Gly-OH 4d, 65 & $200-202$ \\
5 & $\mathbf{2 b}$ & AO-Ala-OH 3b & 4-Mpaba-AO-Ala-OH 4e, 65 & $182-184$ \\
6 & $\mathbf{2 b}$ & AO-Phe-OH 3c & 4-Mpaba-AO-Phe-OH 4f, 75 & $192-194$ \\
7 & $\mathbf{2 c}$ & AO-Gly-OH 3a & 4-Dpaba-AO-Gly-OH 4g, 80 & $189-191$ \\
8 & $\mathbf{2 c}$ & AO-Ala-OH 3b & 4-Dpaba-AO-Ala-OH 4h, 70 & $196-198$ \\
9 & $\mathbf{2 c}$ & AO-Phe-OH 3c & 4-Dpaba-AO-Phe-OH 4i, 80 & $222-224$ \\
\hline
\end{tabular}

an our aminoxy acid nomenclature; the aminoxy acids are defined as follows: "2-(aminoxy)acetic acid 3a" as "AO-Gly-OH 3a"; "2-(aminoxy)propanoic acid 3b" as "AO-Ala-OH 3b"; "2(aminoxy)-3-phenylpropanoic acid 3c" as "AO-Phe-OH 3c" and the abbreviated short names are used throughout the manuscript. ${ }^{\mathrm{b}}$ Isolated yield.

\section{Preparation of Fmoc-protected aminoxy hybrid peptides $(6 a-e)$}

Fmoc protected aminoxy hybrid peptides $6 \mathbf{a}-\mathbf{e}$ were prepared by the reaction of $N$-(Fmoc- $\alpha-$ aminoacyl)benzotriazoles $\mathbf{5 a}-\mathbf{c}$ with aminoxy acids $\mathbf{3 a}, \mathbf{b}$ in the presence of triethylamine in 
acetonitrile-water (3:1) at room temperature in 90-92\% yields (Scheme 2, Table 2).<smiles>[R]C(N)C(=O)Br</smiles>

5a-c<smiles>[R]C(ON)C(=O)O</smiles>

3a, $\mathrm{R}^{2}=\mathrm{H}$

$3 b, \mathrm{R}^{2}=\mathrm{CH}_{3}$

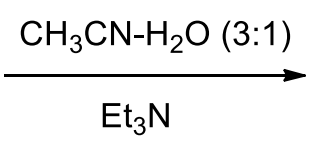

$\mathrm{Et}_{3} \mathrm{~N}$<smiles></smiles>

6a-e

\section{Scheme 2}

Table 2. Preparation of Fmoc-protected aminoxy hybrid peptides $\mathbf{6 a}-\mathbf{e}$

\begin{tabular}{ccccc}
\hline Entry & $\mathbf{3}$ & $\mathbf{5}$ & $\mathbf{6}$, yield $(\%)^{\mathrm{a}}$ & $\mathbf{6}, \mathrm{Mp}^{\circ} \mathrm{C}$ \\
\hline 1 & $\mathbf{3 a}$ & Fmoc-L-Phe-Bt 5b & Fmoc-L-Phe-AO-Gly-OH 6a, 92 & $106-108$ \\
2 & $\mathbf{3 b}$ & Fmoc-L-Phe-Bt 5b & Fmoc-L-Phe-AO-Ala-OH 6b, 90 & $102-104$ \\
3 & $\mathbf{3 a}$ & Fmoc-L-Met-Bt 5c & Fmoc-L-Met-AO-Gly-OH 6c, 92 & $70-72$ \\
4 & $\mathbf{3 a}$ & Fmoc-L-Leu-Bt 5a & Fmoc-L-Leu-AO-Gly-OH 6d, 90 & $110-112$ \\
5 & $\mathbf{3 b}$ & Fmoc-L-Met-Bt 5c & Fmoc-L-Met-AO-Ala-OH 6e, 91 & $88-90$ \\
\hline
\end{tabular}

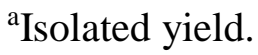

\section{Preparation of azodye labeled aminoxy peptides (7a-c)}

Fmoc protected aminoxy dipeptides $\mathbf{6 a}-\mathbf{c}$ were treated with a solution of one equivalent of DBU in dry THF for $2 \mathrm{~h}$ at $0-5{ }^{\circ} \mathrm{C}$, followed by treatment with 4-phenylazobenzoyl- $1 H$-benzotriazole $2 \mathbf{a}$ gave azodye labeled aminoxy peptides $7 \mathbf{a}-\mathbf{c}$ in $55-65 \%$ yields (Scheme 3, Table 3).

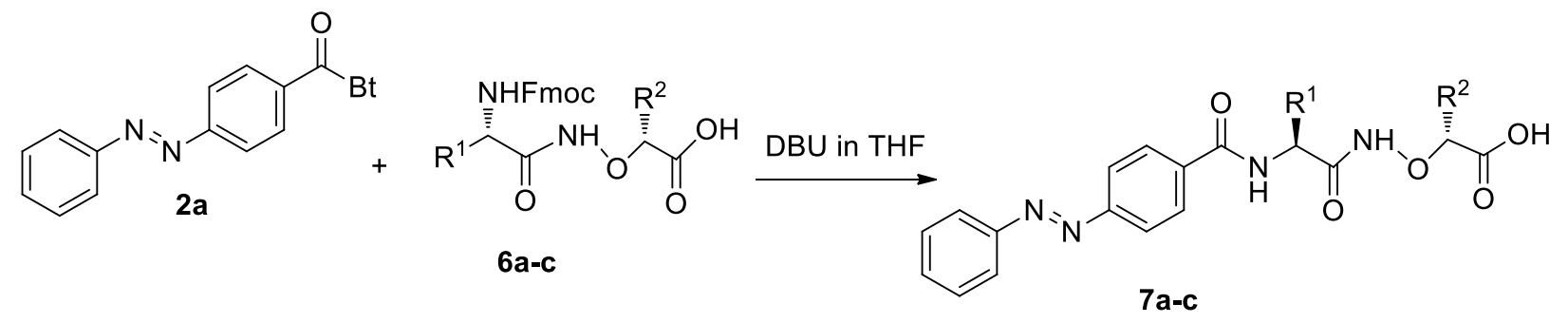

\section{Scheme 3}

Table 3. Preparation of azodye carboxylic acid labeled aminoxy peptides $7 \mathbf{a}-\mathbf{c}$

\begin{tabular}{cccr}
\hline Entry & Fmoc aminoxy dipeptide 6 & 7, yield (\%) & 7, Mp ${ }^{\circ} \mathrm{C}$ \\
\hline 1 & Fmoc-L-Phe-AO-Gly-OH 6a & 4-Paba-L-Phe-AO-Gly-OH 7a, 55 & $178-180$ \\
2 & Fmoc-L-Phe-AO-Ala-OH 6b & 4-Paba-L-Phe-AO-Ala-OH 7b, 58 & $192-194$ \\
3 & Fmoc-L-Met-AO-Gly-OH 6c & 4-Paba-L-Met-AO-Gly-OH 7c, 65 & $185-187$ \\
\hline
\end{tabular}




\section{Conclusions}

In conclusion, we have synthesized novel azodye labeled aminoxy acids and aminoxy hybrid peptides in a convenient and efficient manner by reacting $N$-(4-arylazobenzoyl)- $1 \mathrm{H}$-benzotriazoles with aminoxy acids and aminoxy hybrid peptides. All the azodye labeled aminoxy acids and aminoxy hybrid peptides were obtained under mild reaction conditions in moderate to good yields. These novel azodye labeled aminoxy acids and aminoxy hybrid peptides may be useful in the preparation of peptidomimetic foldamers and in biological applications.

\section{Experimental Section}

General. Aminooxy acid 3a was purchased commercially and used without purification. Aminooxy acids $3 \mathbf{b}, \mathbf{3} \mathbf{c}$ were prepared according to our previously reported method. ${ }^{23}$ Melting points were determined on a capillary point apparatus equipped with a digital thermometer and are uncorrected. NMR spectra were recorded in acetone- $d_{6}, \mathrm{CDCl}_{3}$ or DMSO- $d_{6}$ with TMS for ${ }^{1} \mathrm{H}(300 \mathrm{MHz})$ and ${ }^{13} \mathrm{C}$ $(75 \mathrm{MHz})$ as an internal reference. Elemental analyses were performed on a Carlo Erba-1106 instrument. Mass spectrometry was done on Agilent 6210 TOF-MS with electro spray ionization (ESI). $\mathrm{CH}_{2} \mathrm{Cl}_{2}$ was dried and distilled over $\mathrm{CaH}_{2}$, whereas THF was used after distillation over Nabenzophenone.

\section{Synthesis of azo-dye carboxylic acid labeled aminooxy acids (4a-i)}

$N$-(4-Arylazobenzoyl)- $1 H$-benzotriazoles 2 a-c $(1.0 \mathrm{mmol})$ were added to a solution of the appropriate $\alpha$-aminooxy acids 3a-c $(1.2 \mathrm{mmol})$ in $\mathrm{THF}-\mathrm{H}_{2} \mathrm{O}(3: 1)$ in the presence of $\mathrm{Et}_{3} \mathrm{~N}(2.0$ mmol). The reaction mixture was stirred at $20{ }^{\circ} \mathrm{C}$ for about $3 \mathrm{~h}$ until TLC showed the absence of 2ac, then the solvent was removed under reduced pressure. The residue was dissolved in EtOAc (50 $\mathrm{mL})$ and the solution was washed with $4 \mathrm{~N} \mathrm{HCl}(3 \times 50 \mathrm{~mL})$, saturated $\mathrm{NaCl}$ solution $(50 \mathrm{~mL})$, and dried over anhydrous $\mathrm{Na}_{2} \mathrm{SO}_{4}$. After evaporation of the solvent, the residue was crystallized from appropriate solvents to give $\mathbf{4 a - i}$.

4-Phenylazobenzoyl-AO-Gly-OH (4a). Orange microcrystals (74\%), mp 200-202 ${ }^{\circ} \mathrm{C} .{ }^{1} \mathrm{H}$ NMR $\left(\right.$ DMSO- $\left.d_{6}\right) \delta 8.10-7.93(\mathrm{~m}, 6 \mathrm{H}), 7.70-7.60(\mathrm{~m}, 3 \mathrm{H}), 4.54(\mathrm{~s}, 2 \mathrm{H}) ;{ }^{13} \mathrm{C}$ NMR (DMSO- $\left.d_{6}\right) \delta 171.3$, 163.8 153.8, 152.2, 134.6, 132.5, 130.0, 128.8, 123.1, 122.9, 72.5. Anal. Calcd for $\mathrm{C}_{15} \mathrm{H}_{15} \mathrm{~N}_{3} \mathrm{O}_{5}$ : $\mathrm{C}$, 56.78; H, 4.76; N, 13.24. Found: C, 56.72; H, 4.68; N, 12.94.

4-Phenylazobenzoyl-AO-Ala-OH (4b). Orange microcrystals $(75 \%)$, mp $188-190{ }^{\circ} \mathrm{C} ;[\alpha]^{23} \mathrm{D}=-$ $12.0\left(c 1.0, \mathrm{CH}_{3} \mathrm{OH}\right) .{ }^{1} \mathrm{H}$ NMR (DMSO- $\left.d_{6}\right) \delta 12.05(\mathrm{br} \mathrm{s}, 1 \mathrm{H}), 8.15(\mathrm{~d}, J=8.1 \mathrm{~Hz}, 1 \mathrm{H}), 7.97-7.91$ $(\mathrm{m}, 6 \mathrm{H}), 7.60(\mathrm{~d}, J=1.8 \mathrm{~Hz}, 3 \mathrm{H}), 4.57(\mathrm{q}, J=6.5 \mathrm{~Hz}, 1 \mathrm{H}), 1.43(\mathrm{~d}, J=6.9 \mathrm{~Hz}, 3 \mathrm{H}) ;{ }^{13} \mathrm{C}$ NMR (DMSO- $d_{6}$ ) $\delta 172.7,166.7,151.9,132.1,130.6,129.6,128.7,122.8,122.6,122.5,78.7,16.5$. Anal. Calcd for $\mathrm{C}_{16} \mathrm{H}_{15} \mathrm{~N}_{3} \mathrm{O}_{4}$ : C, 61.34; H, 4.83; N, 13.41. Found: C, 61.37; H, 4.91; N, 13.24. 
4-Phenylazobenzoyl-AO-Phe-OH (4c). Orange microcrystals $(70 \%)$, mp $178-180{ }^{\circ} \mathrm{C} ;[\alpha]^{23} \mathrm{D}=-$ $19.0\left(c\right.$ 1.0, $\left.\mathrm{CH}_{3} \mathrm{OH}\right) .{ }^{1} \mathrm{H}$ NMR (Acetone- $\left.d_{6}\right) \delta 8.05-7.94(\mathrm{~m}, 6 \mathrm{H}), 7.63-7.58(\mathrm{~m}, 3 \mathrm{H}), 7.40(\mathrm{~d}, J=$ $7.2 \mathrm{~Hz}, 2 \mathrm{H}), 7.33-7.23(\mathrm{~m}, 3 \mathrm{H}), 4.93(\mathrm{t}, J=5.1 \mathrm{~Hz}, 1 \mathrm{H}), 3.35-3.20(\mathrm{~m}, 2 \mathrm{H}) ;{ }^{13} \mathrm{C}$ NMR (Acetone- $d_{6}$ ) $\delta 172.0,155.2$, 153.3, 137.5, 134.1, 132.8, 131.6, 130.5, 130.2, 129.3, 129.1, 127.4, 123.8, 123.5, 85.7, 37.9. Anal. Calcd for $\mathrm{C}_{22} \mathrm{H}_{19} \mathrm{~N}_{3} \mathrm{O}_{4}$ : C, 67.86; H, 4.92; N, 10.79. Found: C, 67.62; H, 4.90; N, 10.79 .

4-[(4-Methoxy)phenylazo]benzoyl-AO-Gly-OH (4d). Orange microcrystals (65\%), mp 200-202 ${ }^{\circ} \mathrm{C}$. ${ }^{1} \mathrm{H}$ NMR (DMSO- $\left.d_{6}\right) \delta 12.15$ (br s, $\left.1 \mathrm{H}\right), 8.11(\mathrm{~d}, J=8.1 \mathrm{~Hz}, 1 \mathrm{H}), 7.95-7.86(\mathrm{~m}, 6 \mathrm{H}), 7.15-7.13$ $(\mathrm{m}, 2 \mathrm{H}), 4.52(\mathrm{~s}, 2 \mathrm{H}), 3.87(\mathrm{~s}, 3 \mathrm{H}) ;{ }^{13} \mathrm{C}$ NMR (DMSO- $\left.d_{6}\right) \delta 170.1,162.5146 .2,130.6,128.6,124.9$, 122.3, 122.2, 114.7, 71.8, 55.8. HRMS (ESI) Calcd for $\mathrm{C}_{16} \mathrm{H}_{15} \mathrm{~N}_{3} \mathrm{O}_{5}:[\mathrm{M}-\mathrm{H}]^{-}$328.0939. Found: 328.0938 .

4-[(4-Methoxy)phenylazo]benzoyl-AO-Ala-OH (4e). Orange microcrystals (65\%), mp 182-184 ${ }^{\circ} \mathrm{C} ;[\alpha]^{23} \mathrm{D}=-6.0\left(c 1.0, \mathrm{CH}_{3} \mathrm{OH}\right) .{ }^{1} \mathrm{H}$ NMR (DMSO-d $) \delta 12.03($ br s, $1 \mathrm{H}), 8.12(\mathrm{~d}, J=8.7 \mathrm{~Hz}, 1 \mathrm{H})$, 7.96-7.87 (m, 5H), 7.14 (d, $J=8.1 \mathrm{~Hz}, 2 \mathrm{H}), 6.88$ (br s, 1H), 4.56 (d, J = 6.9 Hz, 1H), 3.87 (s, 3H), $1.42(\mathrm{~d}, J=6.9 \mathrm{~Hz}, 3 \mathrm{H}) ;{ }^{13} \mathrm{C}$ NMR (DMSO-d $) \delta 174.1,163.4,154.7,147.0,133.6,129.3,125.9$, 123.1, 115.5, 79.8, 56.2, 17.1. Anal. Calcd for $\mathrm{C}_{17} \mathrm{H}_{17} \mathrm{~N}_{3} \mathrm{O}_{5}$ : C, 59.47; H, 4.99; N, 12.24. Found: $\mathrm{C}$, $59.13 ; \mathrm{H}, 4.86 ; \mathrm{N}, 12.08$.

4-[(4-Methoxy)phenylazo]benzoyl-AO-Phe-OH (4f). Orange microcrystals (75\%), mp 192-194 ${ }^{\circ} \mathrm{C} ;[\alpha]^{23} \mathrm{D}=-31.0\left(c\right.$ 1.0, $\left.\mathrm{CH}_{3} \mathrm{OH}\right) .{ }^{1} \mathrm{H}$ NMR (Acetone- $\left.d_{6}\right) \delta 11.53$ (br s, $\left.1 \mathrm{H}\right), 8.03-7.92(\mathrm{~m}, 7 \mathrm{H})$, 7.42-7.25 (m, 5H), $7.14(\mathrm{~d}, J=9.0 \mathrm{~Hz}, 2 \mathrm{H}), 4.98-4.93(\mathrm{~m}, 1 \mathrm{H}), 3.93(\mathrm{~s}, 3 \mathrm{H}), 3.37-3.17(\mathrm{~m}, 2 \mathrm{H}) ;{ }^{13} \mathrm{C}$ NMR (Acetone- $\left.d_{6}\right) \delta 171.6,167.6,155.7,147.7,137.4,133.2,130.5,129.5,129.1,127.5,125.9$, 123.2, 115.4, 86.6, 56.1, 38.0. Anal. Calcd for $\mathrm{C}_{23} \mathrm{H}_{21} \mathrm{~N}_{3} \mathrm{O}_{5}$ : C, 65.86; H, 5.05; N, 10.02. Found: C, $65.53 ; \mathrm{H}, 5.08 ; \mathrm{N}, 9.84$.

4-[(4-Dimethylamino)phenylazo]benzoyl-AO-Gly-OH (4g). Brown microcrystals (80\%), mp 189-191 ${ }^{\circ} \mathrm{C} .{ }^{1} \mathrm{H}$ NMR (DMSO-d $) \delta 7.92-7.79(\mathrm{~m}, 7 \mathrm{H}), 6.88(\mathrm{~d}, J=8.4 \mathrm{~Hz}, 2 \mathrm{H}), 4.52(\mathrm{~s}, 2 \mathrm{H}), 3.08$ $(\mathrm{s}, 6 \mathrm{H}) ;{ }^{13} \mathrm{C}$ NMR (DMSO-d $) \delta 170.1,163.7,153.2,142.5,131.8,128.5,126.3,121.3,113.0,71.8$, 40.5. Anal. Calcd for $\mathrm{C}_{17} \mathrm{H}_{19} \mathrm{ClN}_{4} \mathrm{O}_{4}$ : C, 53.90; H, 5.06; N, 14.79. Found: C, 53.78; H, 5.01; N, 14.53.

4-[(4-Dimethylamino)phenylazo]benzoyl-AO-Ala-OH (4h). Brown microcrystals (70\%), $\mathrm{mp}$ $196-198{ }^{\circ} \mathrm{C} ;[\alpha]^{23} \mathrm{D}=-7.0\left(c\right.$ 1.0, $\left.\mathrm{CH}_{3} \mathrm{OH}\right) .{ }^{1} \mathrm{H}$ NMR (DMSO- $\left.d_{6}\right) \delta 8.01-7.95(\mathrm{~m}, 2 \mathrm{H}), 7.90-7.80(\mathrm{~m}$, $4 \mathrm{H}), 6.9(\mathrm{~d}, J=9.0 \mathrm{~Hz}, 2 \mathrm{H}), 4.63(\mathrm{q}, J=7.2 \mathrm{~Hz}, 1 \mathrm{H}), 3.13(\mathrm{~s}, 6 \mathrm{H}), 1.47(\mathrm{~d}, J=6.9 \mathrm{~Hz}, 3 \mathrm{H}) ;{ }^{13} \mathrm{C}$ NMR (DMSO- $\left.d_{6}\right) \delta 173.3,164.7,154.1,153.5,142.8,132.1,128.8,126.2,121.9,112.6,79.2,40.5$, 16.9. Anal. Calcd for $\mathrm{C}_{18} \mathrm{H}_{21} \mathrm{ClN}_{4} \mathrm{O}_{4}$ : C, 55.03; H, 5.39; N, 14.26. Found: C, 54.78; H, 5.51; N, 13.99.

4-[(4-Dimethylamino)phenylazo]benzoyl-AO-Phe-OH (4i). Brown microcrystals (80\%), mp 222$224{ }^{\circ} \mathrm{C} ;[\alpha]^{23} \mathrm{D}=-24.0\left(c\right.$ 1.0, $\left.\mathrm{CH}_{3} \mathrm{OH}\right) .{ }^{1} \mathrm{H}$ NMR (DMSO- $\left.d_{6}\right) \delta 8.00-7.85(\mathrm{~m}, 7 \mathrm{H}), 7.40-7.21(\mathrm{~m}$, $5 \mathrm{H}), 7.03(\mathrm{~d}, J=9.0 \mathrm{~Hz}, 2 \mathrm{H}), 4.85(\mathrm{t}, J=6.6 \mathrm{~Hz}, 1 \mathrm{H}), 3.22-3.08(\mathrm{~m}, 8 \mathrm{H}) ;{ }^{13} \mathrm{C}$ NMR (DMSO- $\left.d_{6}\right) \delta$ 171.6, 153.8, 153.2, 136.7, 132.1, 129.7, 128.6, 128.2, 126.6, 126.2, 121.5, 113.2, 83.7, 45.5, 37.0. Anal. Calcd for $\mathrm{C}_{24} \mathrm{H}_{25} \mathrm{ClN}_{4} \mathrm{O}_{4}$ : C, 61.47; H, 5.37; N, 11.95. Found: C, 60.69; H, 5.42; N, 11.64.

\section{Synthesis of Fmoc-protected aminooxy peptides (6a-e)}


Benzotriazole derivatives of Fmoc-amino acids 5a-c $(1 \mathrm{mmol})$ were added to a solution of the appropriate $\alpha$-aminooxy acids 3a-b $(1 \mathrm{mmol})$ in $\mathrm{MeCN}-\mathrm{H}_{2} \mathrm{O}(3: 1)$ in the presence of $\mathrm{Et}_{3} \mathrm{~N}(2.0$ mmol). The reaction mixtures were stirred at $20{ }^{\circ} \mathrm{C}$ for about $1 \mathrm{~h}$ until TLC showed the absence of 5a-c when the solvent was removed under reduced pressure. The residue was dissolved in EtOAc $(150 \mathrm{~mL})$ and the solution was washed with $4 \mathrm{~N} \mathrm{HCl}(3 \times 50 \mathrm{~mL})$, saturated $\mathrm{NaCl}$ solution $(50 \mathrm{~mL})$, and dried over anhydrous $\mathrm{Na}_{2} \mathrm{SO}_{4}$. After evaporation of the solvent, the residue was crystallized from appropriate solvents to give 6a-e. Compounds 6a-c were used for reaction without further purifications.

Fmoc-L-Phe-AO-Gly-OH (6a). White microcrystals (92\%), mp 106-108 ${ }^{\circ} \mathrm{C} ;[\alpha]^{23} \mathrm{D}=-24.0(c) 1.0$, $\left.\mathrm{CH}_{3} \mathrm{OH}\right) .{ }^{1} \mathrm{H}$ NMR (Acetone- $\left.d_{6}\right) \delta 7.85(\mathrm{~d}, J=7.2 \mathrm{~Hz}, 2 \mathrm{H}), 7.68-7.61(\mathrm{~m}, 2 \mathrm{H}), 7.41(\mathrm{t}, J=7.2 \mathrm{~Hz}$, 2H), 7.34-7.20 (m, 7H), 4.40 (br s, 2H), 4.28-4.16 (m, 3H), 3.22-2.90 (m, 2H); ${ }^{13} \mathrm{C}$ NMR (Acetone$\left.d_{6}\right) \delta 154.6,151.7,147.6,139.9,138.9,138.2,137.6,137.2,135.8,130.4,83.7,77.0,57.5,48.2$, 40.3, 29.6. Anal. Calcd for $\mathrm{C}_{26} \mathrm{H}_{24} \mathrm{~N}_{2} \mathrm{O}_{6}$ : C, 67.82; H, 5.25; N, 6.08. Found: : C, 67.85; H, 5.49; N, 5.55

Fmoc-L-Phe-AO-Ala-OH (6b). White microcrystals (90\%), mp 102-104 ${ }^{\circ} \mathrm{C} ;[\alpha]^{23} \mathrm{D}=-30.0(c$ 1.0, $\left.\mathrm{CH}_{3} \mathrm{OH}\right) .{ }^{1} \mathrm{H} \mathrm{NMR}\left(\mathrm{CDCl}_{3}\right) \delta 10.04$ (br s, 1H), $7.74(\mathrm{~d}, J=7.5 \mathrm{~Hz}, 2 \mathrm{H}), 7.60-7.00(\mathrm{~m}, 12 \mathrm{H}), 5.77$ (br s, $1 \mathrm{H}), 4.63-4.12(\mathrm{~m}, 4 \mathrm{H}), 3.03(\mathrm{~d}, J=7.2 \mathrm{~Hz}, 2 \mathrm{H}), 1.44(\mathrm{~s}, 3 \mathrm{H}) ;{ }^{13} \mathrm{C} \mathrm{NMR}\left(\mathrm{CDCl}_{3}\right) \delta 171.5$, 169.1, 156.4, 143.3, 141.2, 135.4, 129.2, 128.7, 127.8, 127.3, 127.1, 124.9, 120.0, 72.8, 67.7, 60.4, 53.4, 46.8, 38.8. Anal. Calcd for $\mathrm{C}_{27} \mathrm{H}_{26} \mathrm{~N}_{2} \mathrm{O}_{6}$ : C, 68.34; H, 5.52; N, 5.90. Found: C, 67.85; H, 5.49; N, 5.55 .

Fmoc-L-Met-AO-Gly-OH (6c). White microcrystals $(92 \%), \mathrm{mp} 70-72{ }^{\circ} \mathrm{C} ;[\alpha]^{23} \mathrm{D}=-18.0(c$ 1.0, $\left.\mathrm{CH}_{3} \mathrm{OH}\right) .{ }^{1} \mathrm{H}$ NMR $\left(\mathrm{CDCl}_{3}\right) \delta 10.32($ br s, $1 \mathrm{H}), 7.75(\mathrm{~d}, J=7.5 \mathrm{~Hz}, 2 \mathrm{H}), 7.60-7.50(\mathrm{~m}, 2 \mathrm{H}), 7.42-$ 7.25 (m, 6H), 4.51 (s, 2H), 4.50-4.38 (br s, 3H), 4.22-4.18 (m, 1H), 2.60-2.40 (m, 2H), 2.20-1.80 $(\mathrm{m}, 5 \mathrm{H}) ;{ }^{13} \mathrm{C}$ NMR (Acetone-d6) $\delta 173.7,170.4,157.0,145.0,142.0,128.5,127.9,126.1,120.8$, 74.1, 67.3, 67.1, 53.7, 52.7, 47.9, 32.2, 32.1, 30.9, 15.1. Anal. Calcd for $\mathrm{C}_{22} \mathrm{H}_{24} \mathrm{~N}_{2} \mathrm{O}_{6} \mathrm{~S}: \mathrm{C}, 59.45 ; \mathrm{H}$, 5.44; N, 6.30. Found: C, 60.44; H, 5.53; N, 5.89. Compound $\mathbf{6 c}$ was used for the further reaction and the final compound $7 \mathrm{c}$ was fully characterized by ${ }^{1} \mathrm{H},{ }^{13} \mathrm{C}$ NMR and HRMS.

Fmoc-L-Leu-AO-Gly-OH (6d). White microcrystals (90\%), mp 110-112 ${ }^{\circ} \mathrm{C} ;[\alpha]^{23} \mathrm{D}=-14.0(c$ 1.0, $\left.\mathrm{CH}_{3} \mathrm{OH}\right) .{ }^{1} \mathrm{H}$ NMR $\left(\mathrm{CDCl}_{3}\right) \delta 10.54($ br s, $1 \mathrm{H}), 7.73(\mathrm{~d}, J=7.5 \mathrm{~Hz}, 2 \mathrm{H}), 7.60-7.45(\mathrm{~m}, 2 \mathrm{H}), 7.41-$ $7.25(\mathrm{~m}, 5 \mathrm{H}), 5.69(\mathrm{~d}, J=9.6 \mathrm{~Hz}, 1 \mathrm{H}), 4.50(\mathrm{~s}, 2 \mathrm{H}), 4.43-4.30(\mathrm{~m}, 3 \mathrm{H}), 4.15(\mathrm{t}, J=6.0 \mathrm{~Hz}, 1 \mathrm{H})$, $1.57(\mathrm{~d}, J=3.9 \mathrm{~Hz}, 3 \mathrm{H}), 0.89(\mathrm{~d}, J=4.5 \mathrm{~Hz}, 6 \mathrm{H}) ;{ }^{13} \mathrm{C} \mathrm{NMR}\left(\mathrm{CDCl}_{3}\right) \delta 171.6,170.1,156.7,143.3$, 141.3, 127.8, 127.1, 124.9, 120.0, 72.8, 67.7, 50.4, 46.9, 41.5, 24.5, 22.6, 22.0. Anal. Calcd for $\mathrm{C}_{23} \mathrm{H}_{26} \mathrm{~N}_{2} \mathrm{O}_{6}$ : C, 64.78; H, 6.14; N, 6.57. Found: C, 64.63; H, 5.87; N, 6.61.

Fmoc-L-Met-AO-Ala-OH (6e). White microcrystals $(91 \%), \mathrm{mp} 88-90{ }^{\circ} \mathrm{C}$; $[\alpha]^{23}{ }_{\mathrm{D}}=-16.0(c)$, $\left.\mathrm{CH}_{3} \mathrm{OH}\right) .{ }^{1} \mathrm{H} \mathrm{NMR}\left(\mathrm{CDCl}_{3}\right) \delta 10.98$ (br s, $\left.1 \mathrm{H}\right), 7.84(\mathrm{~d}, J=7.8 \mathrm{~Hz}, 2 \mathrm{H}), 7.75-7.60(\mathrm{~m}, 2 \mathrm{H}), 7.45-$ $7.25(\mathrm{~m}, 6 \mathrm{H}), 4.52(\mathrm{~d}, J=6.9 \mathrm{~Hz}, 1 \mathrm{H}), 4.40-4.20(\mathrm{~m}, 4 \mathrm{H}), 2.60-2.51(\mathrm{~m}, 3 \mathrm{H}), 2.07-2.02(\mathrm{~m}, 4 \mathrm{H})$, $1.41(\mathrm{~d}, J=6.9 \mathrm{~Hz}, 3 \mathrm{H}) ;{ }^{13} \mathrm{C} \mathrm{NMR}\left(\mathrm{CDCl}_{3}\right) \delta 174.3,169.6,156.5,143.4,141.1,127.7,127.0$, 124.9, 119.9, 79.7, 67.5, 46.7, 31.7, 31.4, 29.7, 16.1, 15.1. Anal. Calcd for $\mathrm{C}_{23} \mathrm{H}_{26} \mathrm{~N}_{2} \mathrm{O}_{6} \mathrm{~S}: \mathrm{C}, 60.25$; H, 5.72; N, 6.11. Found: C, 60.05; H, 5.71; N, 5.99.

\section{Synthesis of azodye labeled aminooxy peptides (7a-c)}


Fmoc protected aminooxy dipeptide 6a-c $(1 \mathrm{mmol})$ were added to a solution of DBU (1 mmol) in dry THF (6 mL) and after stirring for $2 \mathrm{~h}$ at $0-5{ }^{\circ} \mathrm{C}$, 4-Phenylazobenzoyl-1H-benzotriazole $2 \mathrm{a}$ (1 mmol) was added and the reaction mixtures were stirred at $20{ }^{\circ} \mathrm{C}$ for another $10 \mathrm{~h}$. Solvent was evaporated and residue was dissolved in $\mathrm{CH}_{2} \mathrm{Cl}_{2}(20 \mathrm{~mL})$, washed with $3 \mathrm{~N} \mathrm{HCl}(3 \times 10 \mathrm{~mL})$ and dried over anhydrous $\mathrm{Na}_{2} \mathrm{SO}_{4}$. After evaporation of solvent, the residue was recrystalized from $\mathrm{CH}_{2} \mathrm{Cl}_{2} /$ hexanes to give 7a-c.

4-Phenylazobenzoyl-L-Phe-AO-Gly-OH (7a). Orange microcrystals (55\%), mp 178-180 ${ }^{\circ} \mathrm{C} ;[\alpha]^{23} \mathrm{D}$ $=-46.0\left(c\right.$ 1.0, $\left.\mathrm{CH}_{3} \mathrm{OH}\right) .{ }^{1} \mathrm{H}$ NMR (Acetone- $\left.d_{6}\right) \delta 8.02(\mathrm{~d}, J=8.7 \mathrm{~Hz}, 2 \mathrm{H}), 7.99-7.91(\mathrm{~m}, 4 \mathrm{H}), 7.58-$ $7.56(\mathrm{~m}, 3 \mathrm{H}), 7.35-7.17(\mathrm{~m}, 7 \mathrm{H}), 6.85$ (d, $J=8.7 \mathrm{~Hz}, 1 \mathrm{H}), 4.84$ (br s, 1H), 4.40 (br s, 2H), 3.39-3.10 $(\mathrm{m}, 2 \mathrm{H}) ;{ }^{13} \mathrm{C}$ NMR (Acetone- $\left.d_{6}\right) \delta 170.2,155.0,153.4,138.1,136.9,132.7,130.2,129.4,129.2$, 127.5, 123.7, 123.3, 121.1, 120.5, 74.1, 53.7, 38.0. HRMS (ESI) Calcd for $\mathrm{C}_{24} \mathrm{H}_{22} \mathrm{~N}_{4} \mathrm{O}_{5}:[\mathrm{M}-\mathrm{H}]^{-}$ 445.1517. Found: 445.1521.

4-Phenylazobenzoyl-L-Phe-AO-Ala-OH (7b). Orange microcrystals $(58 \%)$, mp 192-194 ${ }^{\circ} \mathrm{C} ;[\alpha]^{23} \mathrm{D}$ $=-65.0\left(c 1.0, \mathrm{CH}_{3} \mathrm{OH}\right) .{ }^{1} \mathrm{H} \mathrm{NMR}\left(\mathrm{DMSO}_{-} d_{6}\right) \delta 12.81(\mathrm{br} \mathrm{s}, 1 \mathrm{H}), 8.79(\mathrm{~d}, J=8.7 \mathrm{~Hz}, 1 \mathrm{H}), 8.53(\mathrm{~d}, J$ $=7.8 \mathrm{~Hz}, 1 \mathrm{H}), 8.15(\mathrm{~d}, J=10.8 \mathrm{~Hz}, 1 \mathrm{H}), 8.04-7.91(\mathrm{~m}, 5 \mathrm{H}), 7.61(\mathrm{~s}, 3 \mathrm{H}), 7.42-7.16(\mathrm{~m}, 5 \mathrm{H}), 4.79$ (br s, $1 \mathrm{H}), 4.26(\mathrm{~d}, J=6.6 \mathrm{~Hz}, 1 \mathrm{H}) 3.20-3.00(\mathrm{~m}, 2 \mathrm{H}), 1.35(\mathrm{~d}, J=6.6 \mathrm{~Hz}, 3 \mathrm{H}) ;{ }^{13} \mathrm{C}$ NMR (DMSO$\left.d_{6}\right) \delta 174.0,171.2,165.4,153.2,151.8,138.4,132.0,130.6,129.5,129.1,128.7,128.0,122.7$, 122.5, 122.2, 54.7, 47.6, 37.1, 17.1. HRMS (ESI) Calcd for $\mathrm{C}_{25} \mathrm{H}_{24} \mathrm{~N}_{4} \mathrm{O}_{5}:\left[\mathrm{M}+\mathrm{Na}^{+} 483.1639\right.$. Found: 483.1645.

4-Phenylazobenzoyl-L-Met-AO-Gly-OH (7c). Orange microcrystals (65\%), mp 185-187 ${ }^{\circ} \mathrm{C}$; $[\alpha]^{23} \mathrm{D}=-32.0\left(c 1.0, \mathrm{CH}_{3} \mathrm{OH}\right) .{ }^{1} \mathrm{H}$ NMR (DMSO- $\left.d_{6}\right) \delta 8.10-8.00(\mathrm{~m}, 2 \mathrm{H}), 7.88-7.82(\mathrm{~m}, 5 \mathrm{H}), 7.55-$ $7.45(\mathrm{~m}, 3 \mathrm{H}), 4.45(\mathrm{~d}, J=5.7 \mathrm{~Hz}, 1 \mathrm{H}), 4.31(\mathrm{~s}, 2 \mathrm{H}), 2.51-2.45(\mathrm{~m}, 4 \mathrm{H}), 2.00-1.94(\mathrm{~m}, 3 \mathrm{H}) ;{ }^{13} \mathrm{C}$ NMR (DMSO- $\left.d_{6}\right) \delta 173.9,170.4,167.0,154.8,153.2,137.1,132.9,130.4,130.1,129.9,123.9$, 123.5, 123.4, 121.8, 118.0, 114.2, 110.4, 71.2, 52.8, 50.9, 50.6, 38.3. HRMS (ESI) Calcd for $\mathrm{C}_{20} \mathrm{H}_{22} \mathrm{~N}_{4} \mathrm{O}_{5} \mathrm{~S}:[\mathrm{M}+\mathrm{Na}]^{+}$453.1203. Found: 453.1215 .

\section{Acknowledgements}

We thank Dr. C. D. Hall for helpful discussions.

\section{References}

1. Renner, C.; Moroder, L. Chem. Bio. Chem. 2006, 7, 868.

2. Willner, I. Acc. Chem. Res. 1997, 30, 347.

3. Abell, A. D.; Jones, M. A.; Neffe, A. T.; Aitken, S. G.; Cain, T. P.; Payne, R. J.; McNabb, S. B.; Coxon, J. M.; Stuart, B. G.; Pearson, D.; Lee, H. Y. -Y.; Morton, J. D. J. Med. Chem. 2007, 50, 2916.

4. Anderson, M. O.; Wu, L. Y.; Santiago, N. M.; Moser, J. M.; Rowley, J. A.; Bolstad, E. S. D.; Berkman, C. E. Bioorg. Med. Chem. 2007, 15, 6678. 
5. Fraga, S. M. B.; Gonçalves, M. S. T.; Moura, J. C. V. P.; Rani, K. Eur. J. Org. Chem. 2004, 8, 1750.

6. (a) Shen, Z.; Zhang,Y. Synth. Commun. 2000, 30, 2525. (b) Wang, G. T.; Chung, C. C.; Holzman, T. F.; Krafft, G. A. Anal. Biochem. 1993, $210,351$.

7. Wu, Y. -D.; Wang, D. -P.; Chan, K. W. K.; Yang, D. J. Am. Chem. Soc. 1999, 121, 11189.

8. $\quad$ Briggs, M. T.; Morley, J. S. J. Chem. Soc., Perkin Trans. 1. 1979, 2138.

9. (a) Hill, D. J.; Mio, M. J.; Prince, R. B.; Hughes, T. S.; Moore, J. S. Chem. Rev. 2001, 101, 3893. (b) Yang, D.; Zhang, D.-W.; Hao, Y.; Wu, Y.-D.; Luo, S.-W.; Zhu, N.-Y. Angew.

Chem. Int. Ed. 2004, 43, 6719. (c) Li, X.; Yang, D. Chem. Commun. 2006, 3367.

10. (a) Jimenez-Castells, C.; De la Torre, B. G.; Gallego, R. G.; Andreu, D. Bioorg. Med. Chem. Lett. 2007, 17, 5155. (b) Carrasco, M. R.; Nguyen, M. J.; Burnell, D. R.; MacLaren, M. D.; Hengel, S. M. Tetrahedron Lett. 2002, 43, 5727. (c) Carrasco, M. R.; Brown, R. T. J. Org. Chem. 2003, 68, 8853.

11. (a) Chandrasekhar, S.; Rao, C. L.; Reddy, M. S.; Sharma, G. D.; Kiran, M. U.; Naresh, P.; Chaitanya, G. K.; Bhanuprakash, K.; Jagadeesh, B. J. Org. Chem. 2008, 73, 9443. (b) Chen, F.; Song, K.-S.; Wu, Y.-D.; Yang, D. J. Am. Chem. Soc. 2008, 130, 743.

12. Katritzky, A. R.; Suzuki, K.; Wang, Z. Synlett. 2005, 1656.

13. Katritzky, A. R.; Angrish, P.; Suzuki, K. Synthesis 2006, 411.

14. Katritzky, A. R.; Suzuki, K.; Singh, S. K.; He, H. -Y. J. Org. Chem. 2003, 68, 5720.

15. Katritzky, A. R.; He, H. -Y.; Suzuki, K. J. Org. Chem. 2000, 65, 8210.

16. Katritzky, A. R.; Wang, M.; Yang, H.; Zhang, S.; Akhmedov, N. G. Arkivoc 2002, (viii), 134.

17. Katritzky, A. R.; Shestopalov, A. A.; Suzuki, K. Arkivoc 2005, (vii), 36.

18. Katritzky, A. R.; Tala, S. R.; Singh, S. K. Synthesis 2006, 3231.

19. Katritzky, A. R.; Belyakov, S.A. Aldrichim. Acta 1998, 31, 35.

20. Katritzky, A. R.; Li, J.; Xie, L. Tetrahedron 1999, 55, 8263.

21. (a) Katritzky, A. R.; Chen, Q. -Y.; Tala, S. R. Org. Biomol. Chem. 2008, 6, 2400. (b) Katritzky, A. R.; Tala, S. R.; Abo-Dya, N. E.; Abdel-Samii, Z. K. Synthesis 2009, 1708.

22. Katritzky, A. R.; Chen, Q. -Y.; Tala, S. R. Chem. Biol. Drug. Design 2009, 73, 611.

23. Katritzky, A. R.; Avan, I.; Tala, S. R. J. Org. Chem. 2009, 74, 8690. 